\title{
Response of Mode of Application with Integrated Nutrient Management on Soil Parameters of Isabgol (Plantago ovata Forsk.) under Northern Dry Zone of Karnataka
}

\author{
Siddalingayya V. Salimath", K. N. Kattimani, Y. K. Kotikal, N. S. Nagarja, Jameel \\ Jhalegar, D. R. Patil and J. Venkatesh
}

College of Horticulture, Bagalkot, University of Horticultural Sciences, Bagalkot, India

*Corresponding author

\begin{tabular}{l} 
Ke y w o r d s \\
Gujarat Isabgol-2, \\
INM, Vallabh \\
Isabgol-1 \\
\hline Article Info \\
$\begin{array}{l}\text { Accepted: } \\
\text { 18 November } 2020 \\
\text { Available Online: } \\
\text { 10 December } 2020\end{array}$ \\
\hline
\end{tabular}

\section{A B S T R A C T}

The field research trial was conducted to evaluate the two Isabgol varieties with INM in northern dry zone of Karnataka during two years 2015 and 2016. The experiment was laid out in split plot design (SPD) with sixteen INM treatment combinations at the college of horticulture, bagalkot. Analysis of both years and pooled data exhibited more value was Vallabh Isabgol-1 in soil parameters viz., EC $\left(\mathrm{dSm}^{-1} 0.28\right), \mathrm{pH}(8.26)$ and OC (1.78), available NPK $\left(263.12,34.63,264.84 \mathrm{~kg} \mathrm{ha}^{-1}\right)$, Uptake N P K (1: $\left.0.34: 0.72 \%\right)$ as compared to Gujarat Isabgol-2. Whereas the higher values with INM treatments with respect to plant growth and development were observed in Viz. $\mathbf{N}_{11}-75 \%$ RD of FYM (7.5 $\left.\mathrm{t} \mathrm{ha}^{-1}\right)+75 \%$ RD of NPK (37.5:18.75:22.50 $\left.\mathrm{kg} \mathrm{ha}^{-1}\right)+$ Azospirillum $\left(5 \mathrm{~kg} \mathrm{ha}^{-1}\right)+$ PSB ( $3 \mathrm{~kg}$ $\left.\mathrm{ha}^{-1}\right)+\mathrm{ZnSO}_{4}\left(15 \mathrm{~kg} \mathrm{ha}^{-1}\right)+\mathrm{FeSO}_{4}\left(7.5 \mathrm{~kg} \mathrm{ha}^{-1}\right)$ and $\mathrm{EC}, \mathrm{pH}$ and OC $(8.25,0.27,1.90)$, Available NPK (279.62:39.90:286.49 $\mathrm{Kg} \mathrm{ha}^{-1}$ ), Uptake N P K (1.25:0.44:0.95 \%). Higher values for interaction effect on growth and yield parameters were recorded in $\mathrm{V}_{1} \mathrm{~N}_{11}$. viz seed yield (15.50 q ha $\left.{ }^{-1}\right)$ husk yield $\left(5.45 \mathrm{q} \mathrm{ha}^{-1}\right)$.

\section{Introduction}

In India, Isabgol occupies an area of 80,000 ha with the total production of 45,000 tonnes and productivity of $0.56 \mathrm{MT} \mathrm{ha}^{-1}$. India is the only country with monopoly in Isabgol production and marketing in the international trade. Thus, with average export volume of 29,000 MT and it earns on an average of ₹ 520 crores annually which contribute to about 80 per cent of the Psyllium traded in the world market. So, it is called as dollar earning medicinal plant of India. Among all the countries in the world, the United States of America is the single major buyer of Psyllium husk from India, accounting for about 75 per cent of the total exports of the material from India (Goraya and Ved, 2019) with annual trade of more than 60,000 MT.

In Karnataka, Isabgol cultivation is very meager and only local cultivars are grown with poor yield. There is also wide yield gap between local cultivars hence high yielding Isabgol varieties used in this research. Performance of any crop or variety depends 
upon nutrient available to crop, soil, organic and inorganic fertilizers use at optimum doses in INM, it adds up to improve soil and nutrient response in terms for plant to synthesis phytosynthates and more uptake of nutrients to plant behind optimal doses has negative impact on plants. Hence, however this study on Isabgol with INM has been carried out with an aim to identify suitable cultivar.

\section{Materials and Methods}

The experiment was conducted in the field at college of horticulture, Bagalkot at haveli farm during the years 2015 and 2016. Geographically, this experimental site was lies in Northern Dry Zone (Zone-3) of Karnataka state, this agro-climatic zone of Karnataka, situated at $16^{\circ}$ North latitude and $74^{\circ} 59^{\prime}$ East longitude and at an altitude of $533.0 \mathrm{~m}$ above mean sea level

The soil of experimental field was red clay loamy in texture, with percentages of sand 22.60 , silt 26.10 and clay 52.20 bulk density $1.25, \mathrm{EC} 0.24 \mathrm{dS} \mathrm{m}^{-1}$ and $\mathrm{pH} 8.22$ (alkaline in reaction) with organic carbon 1.63 and available 268.02, 34.80, $273.69 \mathrm{NPK} \mathrm{kg} \mathrm{ha}{ }^{-1}$. The source of seed collection was DMAPR anandh at Gujarat i.e. Vallabh Isabgol-1( $\left.\mathrm{V}_{1}\right)$ and Gujarat Isabgol-2 $\left(\mathrm{V}_{2}\right)$. Seed were sown in 18 November 2015 and 2016 with gross plot size of $3.6 \mathrm{~m} \mathrm{x} 1.5 \mathrm{~m}=5.40 \mathrm{~m}^{2}$ in split plot design in sixteen INM sub treatments with three replications with subplot listed below.

$\mathbf{N}_{1}$-RDF FYM (10 $\left.\mathrm{t} \mathrm{ha}^{-1}\right)+$ RDF NPK $\left(50: 25: 30 \mathrm{~kg} \mathrm{ha}^{-1}\right)$

$\mathbf{N}_{2}$-RDF FYM $\left(0 \mathrm{t} \mathrm{ha}^{-1}\right)+$ RDF NPK (50:25:30 $\left.\mathrm{kg} \mathrm{ha}^{-1}\right)+\mathrm{ZnSO}_{4}\left(15 \mathrm{~kg} \mathrm{ha}^{-1}\right)$

$\mathbf{N}_{3}$-RDF FYM (10 $\left.\mathrm{t} \mathrm{ha}^{-1}\right)+$ RDF NPK $\left(50: 25: 30 \mathrm{~kg} \mathrm{ha}^{-1}\right)+\mathrm{FeSO}_{4}\left(7.5 \mathrm{~kg} \mathrm{ha}^{-1}\right)$
$\mathbf{N}_{4}$-RDF FYM (10 $\left.\mathrm{t} \mathrm{ha}^{-1}\right)+$ RDF NPK $\left(50: 25: 30 \mathrm{~kg} \mathrm{ha}^{-1}\right)+\mathrm{FeSO}_{4}\left(7.5 \mathrm{~kg} \mathrm{ha}^{-1}\right)+$ $\mathrm{ZnSO}_{4}\left(15 \mathrm{~kg} \mathrm{ha}^{-1}\right)$

$\mathbf{N}_{5}$-Vermicompost $\left(1 \mathrm{t} \mathrm{ha} \mathrm{ha}^{-1}\right)+$ RDF NPK (50:25:30 kg ha $\left.{ }^{-1}\right)$

$\mathbf{N}_{6}$-Vermicompost $\left(1 \mathrm{tha}^{-1}\right)+50 \%$ RDF NPK $\left(50: 25: 30 \mathrm{~kg} \mathrm{ha}^{-1}\right)+$ Azospirillum $\left(5 \mathrm{~kg} \mathrm{ha}^{-1}\right)+$ Azotobacter $\left(5 \mathrm{~kg} \mathrm{ha}^{-1}\right)$

$\mathbf{N}_{7}-75 \% \operatorname{RDF}$ FYM $\left(7.5 \mathrm{t} \mathrm{ha}^{-1}\right)+75 \% \operatorname{RDF}$ NPK (37.5:18.75:22.50 kg ha')

$\mathbf{N}_{8}-75 \%$ RDF FYM (7.5 $\left.\mathrm{t} \mathrm{ha}^{-1}\right)+75 \%$ RDF NPK $\left(37.5: 18.75: 22.50 \mathrm{~kg} \mathrm{ha}^{-1}\right)+$ Azotobacter $\left(5 \mathrm{~kg} \mathrm{ha}^{-1}\right)$

$\mathbf{N}_{9}-75 \%$ RDF FYM $\left(7.5 \mathrm{t} \mathrm{ha}^{-1}\right)+75 \%$ RDF NPK (37.5:18.75:22.50 kg ha $\left.{ }^{-1}\right)+$ Azospirillum $\left(5 \mathrm{~kg} \mathrm{ha}^{-1}\right)$

$\mathbf{N}_{10}-75 \%$ RDF FYM (7.5 $\left.\mathrm{t} \mathrm{ha}^{-1}\right)+75 \%$ RDF NPK (37.5:18.75:22.50 kg ha $\left.{ }^{-1}\right)+P S B(3 \mathrm{~kg}$ $\left.\mathrm{ha}^{-1}\right)$,

$\mathbf{N}_{11}-75 \%$ RDF FYM $\left(7.5\right.$ tha $\left.^{-1}\right)+75 \%$ RDF NPK (37.5:18.75:22.50 kg $\mathrm{ha}^{-}$ $\left.{ }^{1}\right)+$ Azospirillum $\left(5 \mathrm{~kg} \mathrm{ha}{ }^{-1}\right)+$ PSB $\left(3 \mathrm{~kg} \mathrm{ha}{ }^{-}\right.$ $\left.{ }^{1}\right)+\mathrm{ZnSO}_{4}\left(15 \mathrm{~kg} \mathrm{ha}^{-1}\right)+\mathrm{FeSO}_{4}\left(7.5 \mathrm{~kg} \mathrm{ha}^{-1}\right)$

$\mathbf{N}_{12}-50 \%$ RDF FYM $\left(5 \mathrm{tha}^{-1}\right)+50 \%$ RDF NPK $\left(25: 12.5: 15\right.$ NPK kg ha $\left.{ }^{-1}\right)$

$\mathbf{N}_{13}-50 \%$ RD FYM $\left(5 \mathrm{tha}^{-1}\right)+50 \%$ RDF NPK $\left(25: 12.5: 15 \mathrm{~kg} \mathrm{ha}^{-1}\right)+$ Azotobacter $\left(5 \mathrm{~kg} \mathrm{ha}^{-1}\right)$

$\mathbf{N}_{14}-50 \%$ RDF FYM $\left(5 \mathrm{tha}^{-1}\right)+50 \%$ RDF NPK $\left(25: 12.5: 15 \mathrm{~kg} \mathrm{ha}^{-1}\right)+$ Azospirillum $\left(5 \mathrm{~kg} \mathrm{ha}^{-1}\right)$, $\mathbf{N}_{15}-50 \%$ RDF FYM $\left(5 \mathrm{tha}^{-1}\right)+50 \%$ RDF NPK $\left(25: 12.5: 15 \mathrm{~kg} \mathrm{ha}^{-1}\right)+P S B\left(3 \mathrm{~kg} \mathrm{ha}^{-1}\right)$

$\mathbf{N}_{16}-50 \%$ RD FYM $\left(5 \mathrm{tha}^{-1}\right)+75 \%$ RDF NPK (37.5:18.75:22.50 $\left.\mathrm{kg} \mathrm{ha}^{-1}\right)+$ Azospirillum $(5 \mathrm{~kg}$ $\left.\mathrm{ha}^{-1}\right)+P S B \quad\left(3 \mathrm{~kg} \mathrm{ha}{ }^{-1}\right)+\mathrm{ZnSO}_{4} \quad\left(15 \mathrm{~kg} \mathrm{ha}^{-}\right.$ $\left.{ }^{1}\right)+\mathrm{FeSO}_{4}\left(7.5 \mathrm{~kg} \mathrm{ha}^{-1}\right)$. 
Cultural operation followed according to the as per package of practices and taken as however six and seven irrigation were given as per crop requirement.

Five plants were selected randomly in each plot indicated more dry matter production per plants in meter ${ }^{-1}$ row length growth parameter were recorded at the time of harvest in each plot. The grain and straw samples were collected separately from each plot, dried at $60{ }^{0} \mathrm{C}$ for 48 hours. Dry mass was ground in a stainless steel ball mill for nutrient analysis. $\mathrm{N}$ concentration in both seed and straw was estimated by modified Kjeldahal's method (Piper, 1966). The P content was determined using the vanado molybdo phosphoric acid yellow colour method (Jackson 1973), K content was estimated with diacid mixture by using Flame photometer (Stanford, S. and English., 1963). Number of seeds per spike: The seed number was recorded for five spikes from each of five labelled plants from each plot and their average value was taken for the analysis.

\section{Soil analysis}

A composite soil samples was drawn from experimental area after harvesting of the first season Isabgol crop. After words in the same field maize crop was grown soil nutrient exhaustive crop. Then before second year experimentation once again soil samples collected and recorded as initial soil sample for second year before the INM treatment implementation. The representative soil samples were collected at 15 to $30 \mathrm{~cm}$ depth from each treatment. The soil analysed for the different properties are represented as pooled data of two years pertaining on physicochemical properties of soil $\mathrm{pH}, \mathrm{EC}$ and OC and available N P K are presented in Table-I.

The $\mathrm{pH}$ of the soil was recorded by using a simple electrode $\mathrm{pH}$ meter as suggested by
Piper (1966). The soil and water ratio of 1: 2.5 was used for $\mathrm{pH}$ measurement. Electrical conductivity was measured in the wet extract of soil and water suspension (1:2.5) with the help of salubridge (conductivity meter) following the method given by Jackson (1973). Organic carbon percentage in the soil was estimated by the wet acid method described by Jackson (1973). The available nitrogen in the soil was determined by alkaline permanganate method as suggested by Subbaiah and Asija (1956) and expressed in $\mathrm{kg} \mathrm{ha}^{-1}$.

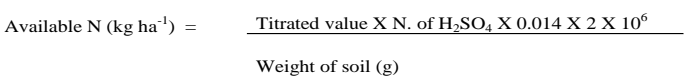

The available phosphorus in the soil was estimated by the colorimetric method as outlined by Olsen et al., (1954) and expressed in $\mathrm{kg}$ ha-1. It is calculated by using the following formula.

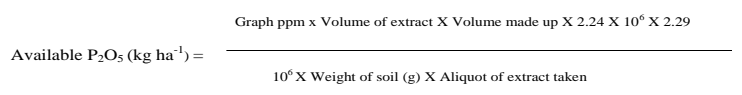

The available potassium was extracted with neutral normal ammonium acetate and the quantity was determined by flame photometer as suggested by Stanford and English (1963) and expressed in $\mathrm{kg} \mathrm{ha}^{-1}$. It is calculated by the formula.

Ivailable $\mathrm{K}_{2} \mathrm{O}\left(\mathrm{kg} \mathrm{ha}^{-1}\right)=$

Graph ppm x Volume of extract X $2.24 \times 10^{6} \times 2.20$

$10^{6} \mathrm{X}$ Weight of soil $(\mathrm{g})$

\section{Plant analysis}

Dried whole plant samples of leaves, stem and roots samples were dried in oven at $650 \mathrm{C}$ for 48 hours and dry weight was recorded, and ground to a fine powder separately, contents of nitrogen, phosphorous and potassium in the plant tissue were analyzed by using suitable methods of analysis. 
The plant samples for NPK analysis were collected at harvesting stage and were dried. The dried leaves were finely grinded in a mixture of a fine powder. This fine powder stored properly in air tight polycarbonate containers with proper labeling till the samples were used for chemical analysis of nutrients.

The total nitrogen (\%) was determined by Kjeldahl method as outlined by Piper (1966). Plant sample of $1 \mathrm{~g}$ each was digested with digestion mixture $\left(\mathrm{CuSO}_{4}, \mathrm{~K}_{2} \mathrm{SO}_{4}\right.$ and $\mathrm{Se}$ in 10:4:1 ratio) in a digestion chamber along with $25 \mathrm{ml}$ of concentrated sulphuric acid. The digested mixture was then distilled and the ammonia released was trapped in four per cent boric acid with mixed indicator. This was titrated against standard sulphuric acid. The total phosphorus (\%) content of the diaciddigested extract was estimated by vanado molybdo-phosphoric acid yellow colour method in nitric acid medium. The intensity of yellow colour was read using spectrophotometer at $420 \mathrm{~nm}$ wave length as described by Piper (1966).

The total potassium content $(\%)$ of the diacid-digested extract was determined by flame photometry as described by Piper (1966). The samples prepared using di-acid or tri acid digested can be fed directly to flame photometry. Dilution should be done before feeding to the instrument (if required). Compare the unknown sample readings with a standard curve to determine the per cent potassium $(\mathrm{K})$ in plant sample. The Uptake of N P K (hg ha $\left.{ }^{-1}\right)$ by Isabgol after harvest was computed by using following formula.

The uptake of nitrogen was calculated by using the formula given below.

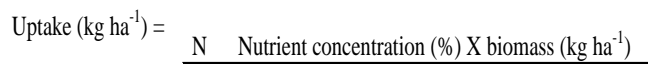

100
The pooled data was subjected to statistical analysis of split-plot design by the Fischer's method of analysis of variance technique as given by Panse and Sukhatme (1983). The level of significance used in ' $F$ ' and ' $t$ ' test was $\mathrm{p}=0.05$, critical values were calculated wherever the ' $F$ ' test was significant.

\section{Results and Discussion}

The data on soil pH, Electric Conductivity $\left(\mathrm{dSm}^{-1}\right)$ lower was recorded in Vallabh Isabgol-1 and higher was recorded in Gujarat Isabgol-2. However, maximum organic carbon was recorded in Vallabh Isabgol-1 as compared to Gujarat Isabgol-2. Available Nitrogen, Phosphorous, Potassium $\left(\mathrm{kg} \mathrm{ha}^{-1}\right)$

In case of Isabgol varieties the available soil Nitrogen, Phosphorous, Potassium (Kg ha $\left.{ }^{-1}\right)$ the minimum was recorded in Vallabh Isabgol-1 as compared to Gujarat Isabgol2.The nutrient content and uptake of nitrogen, phosphorus and potassium maximum was recorded in Vallabh Isabgol-1 as compared to Gujarat Isabgol-2, this might be due to inherent capacity of the genotype and more nutrient uptake results the chemical composition of different parts of the plant. Hence there is a relationship between the nutrient content of plant parts and the absorption of nutrients by the crop during its growth (Solanki et al., 1987 and Sharma and Ratnoo, 2013) (Table 2 and 3).

\section{Integrated nutrient management}

The significantly higher seed yield per hectare in pooled analysis were presented $15.43 \mathrm{q} \mathrm{ha}^{-1}$ was recorded in $\mathrm{N}_{11}$ (75\% RD of FYM +75 $\% \mathrm{RD}$ of $\mathrm{NPK}+$ Azospirillum $+P S B+\mathrm{ZnSO}_{4}$ $+\mathrm{FeSO}_{4}$ ), which were on par with $\mathrm{N}_{6}$ (Vermicompost $+50 \%$ RD of NPK + Azospirillum + Azatobacter) (14.83 q ha $\left.{ }^{-1}\right)$ and $\mathrm{N}_{4}\left(\mathrm{RD}\right.$ of FYM + RD of $\mathrm{NPK}+\mathrm{ZnSO}_{4}+$ $\left.\mathrm{FeSO}_{4}\right)\left(15.12 \mathrm{q} \mathrm{ha}^{-1}\right), \mathrm{N}_{16}(50 \% \mathrm{RD}$ of 
$\mathrm{FYM}+75 \% \mathrm{RD}$ of NPK + Azospirillum + $\left.P S B+\mathrm{ZnSO}_{4}+\mathrm{FeSO}_{4}\right)\left(14.90 \mathrm{q} \mathrm{ha}^{-1}\right)$. Further lower $\left(9.25 \mathrm{q} \mathrm{ha}^{-1}\right)$ seed yield $\mathrm{ha}^{-1}$ was recorded in $\mathrm{N}_{13}(50 \% \mathrm{RD}$ of $\mathrm{FYM}+50 \% \mathrm{RD}$ of NPK+ Azatobacter) during pooled data, which could be due to the increased seed yield consequence with application of balanced nutrient RD of FYM $75 \%+$ RD of NPK micro nutrients mixed with bio fertilizers like azospirillum mechanism through phosphate dissolution and in the biosynthesis of bio-active in soil. The biofertilizers help in fixation of atmospheric nitrogen, better root proliferation, better availability and absorption of nutrients by the plants, $P S B$ helps in reducing phosphorus fixation by its chelating effect and also solubilized the fixed phosphorus accelerated increase in growth of parameters towards reproductive parameters with accelerating tillers, dry matter production, number of spikes per plant, spikelets per plant, spike length, increase towards yield attributing characters viz. number of seeds per spike, ultimately all these growth and reproductive and yield attributes helped to increase seed yield, Similar findings observed by Repsiene (2001), Singh et al., (2011), Tripathi et al., 2013, Choudhary et al., (2014), Nadukeri et al., (2014) and Shivran et al., (2015).

The soil EC was influenced by integrated nutrient management. There was no variation in soil EC with supply of $75 \%$ RD of FYM + $75 \% \mathrm{RD}$ of NPK + Azospirillum + PSB + $\mathrm{ZnSO}_{4}+\mathrm{FeSO}_{4}\left(\mathrm{~N}_{11}\right)$,Vermicompost $+50 \%$ $\mathrm{RD}$ of NPK + Azospirillum + Azotobacter $\left(\mathrm{N}_{6}\right), 50 \% \mathrm{RD}$ of $\mathrm{FYM}+75 \% \mathrm{RD}$ of NPK + Azospirillum $+P S B+\mathrm{ZnSO}_{4}+\mathrm{FeSO}_{4}\left(\mathrm{~N}_{16}\right)$, $\mathrm{RD}$ of $\mathrm{FYM}+\mathrm{RD}$ of $\mathrm{NPK}+\mathrm{ZnSO}_{4}+\mathrm{FeSO}_{4}$ $\left(\mathrm{N}_{4}\right)$ and higher EC was recorded with application of $50 \% \mathrm{RD}$ of FYM + $50 \% \mathrm{RD}$ of NPK + Azotobacter $\left(\mathrm{N}_{13}\right)$. the level of sodium ions has increased but level of potassium ions has decreased considering various growth and development parameters, the assessment of salt tolerance in these treatments could be due to the buffering capacity of soil as affected by the early seed germination, flowering and maturity stages in Isabgol. Similar results reported by Kazal et al., (2017).

The soil $\mathrm{pH}$ influenced by integrated nutrient management. The significantly minimum $\mathrm{pH}$ was recorded with supply of $75 \% \mathrm{RD}$ of $\mathrm{FYM}+75 \% \mathrm{RD}$ of NPK + Azospirillum + $P S B+\mathrm{ZnSO}_{4}+\mathrm{FeSO}_{4}\left(\mathrm{~N}_{11}\right)$ which was followed by Vermicompost $+50 \% \mathrm{RD}$ of $\mathrm{NPK}+$ Azospirillum + Azotobacter $\left(\mathrm{N}_{6}\right), \mathrm{RD}$ of $\mathrm{FYM}+\mathrm{RD}$ of $\mathrm{NPK}+\mathrm{ZnSO}_{4}+\mathrm{FeSO}_{4}\left(\mathrm{~N}_{4}\right)$ and $50 \% \mathrm{RD}$ of $\mathrm{FYM}+75 \% \mathrm{RD}$ of NPK + Azospirillum $+P S B+\mathrm{ZnSO}_{4}+\mathrm{FeSO}_{4}\left(\mathrm{~N}_{16}\right)$. Maximum $\mathrm{pH}$ was recorded with application of RD of FYM + RD of NPK + $\mathrm{FeSO}_{4}\left(\mathrm{~N}_{3}\right)$, Isabgol found optimum soil $\mathrm{EC}, \mathrm{pH}$ and $\mathrm{OC}$ for its growth during all treatments combination which helped easily to available nutrients to plants and uptake of nutrients also than increased growth of plants and this crop can be grown successfully even in moderate alkali soil up to $\mathrm{pH}$ of 9.2, without the application of any soil amendment in semiarid regions of India. It can tolerate high $\mathrm{pH}$ but there was significant yield reduction. Further could be grown successfully with saline water up to EC $12 \mathrm{dS} \mathrm{m}^{-1}$. It results the early seed germination, flowering and maturity stages, enhanced levels of proline, total sugar and soluble protein were observed in tolerant cultivar of Isabgol under stress as reported by Kazal et al., (2017).

The soil organic carbon was influenced by integrated nutrient management. Significant maximum organic carbon was recorded in $\mathrm{N}_{11}$ $(75 \%$ RD of FYM + $75 \%$ RD of NPK + Azospirillum $\left.+P S B+\mathrm{ZnSO}_{4}+\mathrm{FeSO}_{4}\right)$ and least was recorded in $\mathrm{N}_{13}(50 \% \mathrm{RD}$ of FYM $+50 \% \mathrm{RD}$ of NPK + Azotobacter) during both the years and pooled data. 
Table.1 Soil pH, Electric Conductivity and Organic Carbon as influenced by Isabgol varieties and integrated nutrient management after harvest

\begin{tabular}{|c|c|c|c|c|c|c|c|c|c|c|c|c|c|c|c|c|c|c|c|c|c|c|c|c|c|c|c|}
\hline \multirow{3}{*}{$\begin{array}{c}\begin{array}{c}\text { Varieties } \\
\text { Nutrients }\end{array} \\
\text { Nutrients }\end{array}$} & \multicolumn{9}{|c|}{2015} & \multicolumn{9}{|c|}{2016} & \multicolumn{9}{|c|}{ Pooled data } \\
\hline & \multicolumn{3}{|c|}{ Soil pH } & \multicolumn{3}{|c|}{ EC $\left(\mathrm{dSm}^{-1}\right)$} & \multicolumn{3}{|c|}{ Organic carbon $(\%)$} & \multicolumn{3}{|c|}{ Soil pH } & \multicolumn{3}{|c|}{ EC $\left(\mathrm{dSm}^{-1}\right)$} & \multicolumn{3}{|c|}{ Organic carbon (\%) } & \multicolumn{3}{|c|}{ Soil pH } & \multicolumn{3}{|c|}{ EC $\left(\mathrm{dSm}^{-1}\right)$} & \multicolumn{3}{|c|}{ Organic carbon(\%) } \\
\hline & $\mathrm{v}_{\mathrm{i}}$ & $\mathrm{v}_{2}$ & Mean & $\mathrm{v}_{1}$ & $\mathbf{v}_{2}$ & Mean & $v_{i}$ & $\mathbf{v}_{2}$ & Mean & $\mathrm{v}_{\mathrm{i}}$ & $v_{2}$ & Mean & $v_{1}$ & $\mathrm{v}_{2}$ & $\begin{array}{c}\text { Mea } \\
\mathrm{n}\end{array}$ & $v_{1}$ & $\mathrm{~V}_{2}$ & $\begin{array}{c}\text { Mea } \\
\mathrm{n}\end{array}$ & $\mathrm{v}_{\mathbf{1}}$ & $\mathbf{v}_{2}$ & $\begin{array}{c}\text { Mea } \\
\mathrm{n}\end{array}$ & $\mathrm{v}_{\mathbf{1}}$ & $v_{2}$ & $\underset{\mathrm{M}}{\mathrm{Mea}}$ & $v_{1}$ & $\mathrm{v}_{2}$ & Mean \\
\hline$N_{1}$ RDF FYM + RDF NPK (kg ha" & 8.28 & 8.28 & 8.28 & 0.28 & 0.27 & 0.28 & 1.84 & 1.85 & 1.85 & 8.26 & 8.27 & 8.26 & 0.29 & 0.28 & 0.29 & 1.87 & 1.83 & 1.85 & 8.27 & 8.27 & 8.27 & 0.29 & 0.28 & 0.28 & 1.86 & 1.84 & 1.85 \\
\hline$\underset{\text { ZnSO4 }}{\text { N2. }_{2} \text { RDF FYM + RPF NP+ }}$ & 8.27 & 8.31 & 8.29 & 0.28 & 0.27 & 0.28 & 1.84 & 1.83 & 1.84 & 8.27 & 8.24 & 8.26 & 0.29 & 0.28 & 0.28 & 1.86 & 1.84 & 1.85 & 8.27 & 8.28 & 8.27 & 0.28 & 0.28 & 0.28 & 1.85 & 1.84 & 1.84 \\
\hline $\mathrm{N}_{3}-\mathrm{RDF} F \mathrm{FYM}+\mathrm{RDF}$ NPK+FeSO4 & 8.33 & 8.32 & 8.32 & 0.28 & 0.28 & 0.28 & 1.88 & 1.81 & 1.84 & 8.28 & 8.26 & 8.27 & 0.29 & 0.29 & 0.29 & 1.86 & 1.85 & 1.86 & 8.30 & 8.29 & 8.30 & 0.28 & 0.28 & 0.28 & 1.87 & 1.83 & 1.85 \\
\hline $\begin{array}{c}\mathrm{N}_{\mathrm{s}-\text { Vermicompost }}\left(1 \mathrm{t} \mathrm{ha}^{-1}\right)+\mathrm{RDF} \\
\text { NPK }\end{array}$ & 8.26 & 8.25 & 8.26 & 0.27 & 0.27 & 0.27 & 1.89 & 1.87 & 1.88 & 8.25 & 8.27 & 8.26 & 0.28 & 0.26 & 0.27 & 1.89 & 1.89 & 1.89 & 8.26 & 8.26 & 8.26 & 0.28 & 0.26 & 0.27 & 1.89 & 1.88 & 1.89 \\
\hline $\begin{array}{c}\mathbf{N}_{6} \text {-Vermicompost + 50\% RDF } \\
\text { NPK + ASP + AZB }\end{array}$ & 8.26 & 8.25 & 8.26 & 0.27 & 0.27 & 0.27 & 1.89 & 1.89 & 1.89 & 8.28 & 8.24 & 8.26 & 0.27 & 0.27 & 0.27 & 1.90 & 1.89 & 1.90 & 8.27 & 8.25 & 8.26 & 0.27 & 0.27 & 0.27 & 1.90 & 1.89 & 1.89 \\
\hline$\underset{\text { NPK }}{\mathrm{N}_{2} 75 \% \text { RDF FYM }}+75 \%$ RDF & 8.26 & 8.25 & 8.26 & 0.28 & 0.28 & 0.28 & 1.74 & 1.77 & 1.75 & 8.27 & 8.26 & 8.27 & 0.29 & 0.28 & 0.28 & 1.77 & 1.75 & 1.76 & 8.27 & 8.26 & 8.26 & 0.28 & 0.28 & 0.28 & 1.75 & 1.76 & 1.76 \\
\hline 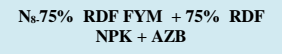 & 8.28 & 8.26 & 8.27 & 0.28 & 0.28 & 0.28 & 1.70 & 1.67 & 1.68 & 8.26 & 8.26 & 8.26 & 0.28 & 0.29 & 0.28 & 1.75 & 1.74 & 1.75 & 8.27 & 8.26 & 8.27 & 0.28 & 0.28 & 0.28 & 1.73 & 1.71 & 1.72 \\
\hline N,75 \% $\underset{\text { NPK }+ \text { ASP }}{\text { RDF FYM }}+75 \%$ RDF & 8.27 & 8.26 & 8.27 & 0.28 & 0.28 & 0.28 & 1.75 & 1.64 & 1.69 & 8.27 & 8.25 & 8.26 & 0.28 & 0.28 & 0.28 & 1.78 & 1.79 & 1.79 & 8.27 & 8.26 & 8.27 & 0.28 & 0.28 & 0.28 & 1.76 & 1.71 & 1.74 \\
\hline 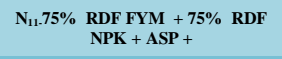 & 8.22 & 8.26 & 8.24 & 0.27 & 0.27 & 0.27 & 1.89 & 1.88 & 1.89 & 8.26 & 8.26 & 8.26 & 0.27 & 0.26 & 0.27 & 1.89 & 1.94 & 1.92 & 8.24 & 8.26 & 8.25 & 0.27 & 0.27 & 0.27 & 1.89 & 1.91 & 1.90 \\
\hline 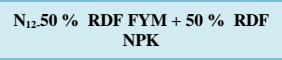 & 8.25 & 8.25 & 8.25 & 0.28 & 0.28 & 0.28 & 1.64 & 1.65 & 1.65 & 8.29 & 8.22 & 8.26 & 0.28 & 0.29 & 0.29 & 1.78 & 1.78 & 1.78 & 8.27 & 8.24 & 8.25 & 0.28 & 0.28 & 0.28 & 1.71 & 1.72 & 1.71 \\
\hline$\underset{\text { NPK }+ \text { AZB }}{\mathrm{N}_{13} 50 \% \text { RDF FYM }}$ & 8.26 & 8.27 & 8.26 & 0.29 & 0.28 & 0.29 & 1.47 & 1.48 & 1.48 & 8.25 & 8.26 & 8.26 & 0.30 & 0.28 & 0.29 & 1.64 & 1.65 & 1.65 & 8.26 & 8.27 & 8.26 & 0.30 & 0.28 & 0.29 & 1.56 & 1.56 & 1.56 \\
\hline 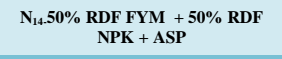 & 8.26 & 8.28 & 8.27 & 0.28 & 0.28 & 0.28 & 1.58 & 1.62 & 1.60 & 8.25 & 8.27 & 8.26 & 0.29 & 0.29 & 0.29 & 1.69 & 1.68 & 1.69 & 8.25 & 8.28 & 8.27 & 0.28 & 0.28 & 0.28 & 1.64 & 1.65 & 1.64 \\
\hline$\underset{\text { NPK + PSB }}{\mathrm{N}_{15} 50 \% \text { Ro\% }}$ & 8.27 & 8.27 & 8.27 & 0.28 & 0.28 & 0.28 & 1.57 & 1.61 & 1.59 & 8.27 & 8.24 & 8.25 & 0.27 & 0.30 & 0.28 & 1.65 & 1.67 & 1.66 & 8.27 & 8.25 & 8.26 & 0.28 & 0.29 & 0.28 & 1.61 & 1.64 & 1.63 \\
\hline 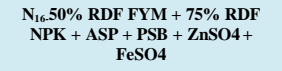 & 8.24 & 8.28 & 8.26 & 0.27 & 0.27 & 0.27 & 1.89 & 1.88 & 1.88 & 8.21 & 8.27 & 8.24 & 0.28 & 0.28 & 0.28 & 1.89 & 1.88 & 1.89 & 8.23 & 8.28 & 8.25 & 0.27 & 0.28 & 0.28 & 1.89 & 1.88 & 1.88 \\
\hline \multirow[t]{2}{*}{ MEAN } & 8.26 & 8.27 & & 0.28 & 0.27 & & 1.76 & 1.75 & & 8.26 & 8.26 & & 0.29 & 0.28 & 0.29 & 1.80 & 1.81 & & 8.26 & 8.26 & & 0.28 & 0.28 & & 1.78 & 1.78 & \\
\hline & S.Em \pm & \multicolumn{2}{|c|}{ C.D at $5 \%$} & $\mathrm{~S} . \mathrm{Em} \pm$ & \multicolumn{2}{|c|}{ C.D at $5 \%$} & $\mathrm{~S} . \mathrm{Em} \pm$ & \multicolumn{2}{|c|}{ C.D at $5 \%$} & S.Em \pm & \multicolumn{2}{|c|}{ C.D at $5 \%$} & S.Em \pm & \multicolumn{2}{|c|}{ C.D at $5 \%$} & $\underset{ \pm}{\text { S.Em }}$ & \multicolumn{2}{|c|}{ C.D at $5 \%$} & $\begin{array}{l}\text { S.E } \\
\mathrm{m} \pm\end{array}$ & \multicolumn{2}{|c|}{ C.D at $5 \%$} & $\begin{array}{l}\text { S.E } \\
\mathrm{m} \pm\end{array}$ & \multicolumn{2}{|c|}{ C.D at $5 \%$} & $\begin{array}{l}\text { S.E } \\
\mathrm{m} \pm\end{array}$ & \multicolumn{2}{|c|}{ C.D at $5 \%$} \\
\hline Varieties (V) & 0.006 & & & 0.002 & & & 0.005 & & & 0.002 & $\mathrm{~N}$ & & 0.002 & & & 0.004 & $\mathrm{~s}$ & & $\begin{array}{r}0.00 \\
4\end{array}$ & Ns & & $\begin{array}{r}0.00 \\
1\end{array}$ & NS & & $\begin{array}{r}0.00 \\
3\end{array}$ & & is \\
\hline Nutrients (N) & 0.014 & & & 0.003 & s & & 0.015 & & & 0.016 & $\mathrm{~N}$ & & 0.004 & & & 0.018 & 0. & & $\begin{array}{r}0.01 \\
2\end{array}$ & Ns & & $\begin{array}{r}0.00 \\
3\end{array}$ & 0.6 & & $\begin{array}{c}0.01 \\
1\end{array}$ & & 03 \\
\hline $\mathrm{N}$ at same $\mathrm{V}$ & 0.020 & & & 0.004 & & & 0.021 & & & 0.023 & $\mathrm{~N}$ & & 0.006 & & & 0.025 & s & & $\begin{array}{r}0.01 \\
8\end{array}$ & Ns & & $\begin{array}{r}0.00 \\
4\end{array}$ & $\mathrm{~N}$ & & $\frac{0.01}{5}$ & & vs \\
\hline
\end{tabular}


Table.2 Available N P and $\mathrm{K}\left(\mathrm{kg} \mathrm{ha}^{-1}\right)$ as influenced by Isabgol varieties and integrated nutrient management

\begin{tabular}{|c|c|c|c|c|c|c|c|c|c|c|c|c|c|c|c|c|c|c|c|c|c|c|c|c|c|c|c|}
\hline \multirow{3}{*}{$\begin{array}{l}\text { Varieties } \\
\text { Nutrients } \\
\text { Nutrients }\end{array}$} & \multicolumn{9}{|c|}{2015} & \multicolumn{9}{|c|}{2016} & \multicolumn{9}{|c|}{ Pooled } \\
\hline & \multicolumn{3}{|c|}{ Available $\mathrm{N}$} & \multicolumn{3}{|c|}{ Available $P_{2} O_{s}$} & \multicolumn{3}{|c|}{ Available $\mathrm{K}_{2} \mathrm{O}$} & \multicolumn{3}{|c|}{ Available $\mathrm{N}$} & \multicolumn{3}{|c|}{ Available $\mathrm{P}_{2} \mathrm{O}_{5}$} & \multicolumn{3}{|c|}{ Available $\mathrm{K}_{2} \mathrm{O}$} & \multicolumn{3}{|c|}{ Available $\mathrm{N}$} & \multicolumn{3}{|c|}{ Available $\mathrm{P}_{2} \mathrm{O}_{5}$} & \multicolumn{3}{|c|}{ Available $\mathrm{K}_{2} \mathrm{O}$} \\
\hline & $v_{1}$ & $\mathbf{v}_{2}$ & Mean & $v_{1}$ & $v_{2}$ & Mean & $\mathrm{v}_{\mathbf{1}}$ & $v_{2}$ & Mean & $v_{1}$ & $\mathbf{v}_{2}$ & Mean & $\mathbf{v}_{\mathbf{i}}$ & $\mathbf{v}_{2}$ & Mean & $v_{1}$ & $\mathbf{v}_{2}$ & Mean & $v_{1}$ & $\mathbf{v}_{2}$ & Mean & $\mathbf{v}_{1}$ & $\mathbf{v}_{2}$ & Mean & $v_{i}$ & $\mathbf{v}_{2}$ & Mean \\
\hline $\mathbf{N}_{\mathbf{t}}$ & 264.22 & 255.55 & 259.89 & 34.93 & 37.00 & 35.97 & 258.72 & 261.42 & 260.07 & 263.09 & 271.75 & 267.42 & 36.43 & 37.17 & 36.80 & 263.22 & 270.59 & 266.91 & 263.65 & 263.65 & 263.65 & 35.68 & 37.09 & 36.38 & 260.97 & 266.01 & 263.49 \\
\hline $\mathrm{N}_{2}$ & 257.79 & 254.79 & 256.29 & 36.31 & 37.27 & 36.79 & 259.39 & 262.09 & 260.74 & 262.32 & 265.32 & 263.82 & 37.81 & 38.77 & 38.29 & 261.89 & 264.93 & 263.41 & 260.05 & 260.05 & 260.05 & 37.06 & 38.02 & 37.54 & 260.64 & 263.51 & 262.08 \\
\hline $\mathrm{N}_{3}$ & 260.49 & 255.16 & 257.82 & 36.57 & 36.98 & 36.77 & 258.20 & 260.12 & 259.16 & 262.69 & 268.02 & 265.36 & 36.74 & 38.48 & 37.61 & 257.36 & 270.28 & 263.82 & 261.59 & 261.59 & 261.59 & 36.66 & 37.73 & 37.19 & 257.78 & 265.20 & 261.49 \\
\hline $\mathbf{N}_{\star}$ & 237.52 & 233.86 & 235.69 & 29.97 & 31.24 & 30.61 & 242.42 & 243.23 & 242.82 & 241.39 & 245.06 & 243.22 & 30.81 & 30.41 & 30.61 & 243.92 & 244.73 & 244.32 & 239.46 & 239.46 & 239.46 & 30.39 & 30.82 & 30.61 & 243.17 & 243.98 & 243.57 \\
\hline $\mathbf{N}_{\mathbf{s}}$ & 253.82 & 247.49 & 250.66 & 35.54 & 35.97 & 35.76 & 267.45 & 268.49 & 267.97 & 255.02 & 261.36 & 258.19 & 37.04 & 37.47 & 37.26 & 268.95 & 269.32 & 269.14 & 254.42 & 254.42 & 254.42 & 36.29 & 36.72 & 36.51 & 268.20 & 268.90 & 268.55 \\
\hline $\mathrm{N}_{6}$ & 238.62 & 235.06 & 236.84 & 29.70 & 32.22 & 30.96 & 244.68 & 244.58 & 244.63 & 242.59 & 246.16 & 244.38 & 30.20 & 30.39 & 30.29 & 245.75 & 246.45 & 246.10 & 240.61 & 240.61 & 240.61 & 29.95 & 31.30 & 30.62 & 245.22 & 245.52 & 245.37 \\
\hline $\mathbf{N}_{7}$ & 253.39 & 255.39 & 254.39 & 32.88 & 35.03 & 33.96 & 250.74 & 262.04 & 256.39 & 252.92 & 260.92 & 256.92 & 34.38 & 36.53 & 35.46 & 251.91 & 263.54 & 257.73 & 253.16 & 258.16 & 255.66 & 33.63 & 35.78 & 34.71 & 251.33 & 262.79 & 257.06 \\
\hline $\mathbf{N}_{\mathbf{s}}$ & 250.96 & 247.96 & 249.46 & 32.80 & 34.88 & 33.84 & 264.12 & 265.82 & 264.97 & 245.49 & 255.16 & 250.32 & 34.30 & 36.38 & 35.34 & 265.62 & 267.32 & 266.47 & 248.22 & 251.56 & 249.89 & 33.55 & 35.63 & 34.59 & 264.87 & 266.57 & 265.72 \\
\hline No & 249.15 & 248.15 & 248.65 & 33.10 & 34.20 & 33.65 & 255.87 & 256.72 & 256.30 & 255.68 & 263.01 & 259.35 & 34.60 & 35.70 & 35.15 & 258.22 & 260.04 & 259.13 & 252.41 & 255.58 & 254.00 & 33.85 & 34.95 & 34.40 & 257.05 & 258.38 & 257.71 \\
\hline $\mathrm{N}_{10}$ & 255.52 & 242.18 & 248.85 & 31.53 & 33.10 & 32.32 & 255.13 & 268.47 & 261.80 & 249.72 & 263.05 & 256.38 & 33.03 & 34.60 & 33.82 & 256.63 & 269.97 & 263.30 & 252.62 & 252.62 & 252.62 & 32.28 & 33.85 & 33.07 & 255.88 & 269.22 & 262.55 \\
\hline $\mathrm{N}_{\mathrm{u}}$ & 234.96 & 229.96 & 232.46 & 29.38 & 28.90 & 29.14 & 241.86 & 242.49 & 242.18 & 237.49 & 242.49 & 239.99 & 29.88 & 30.15 & 30.02 & 242.66 & 244.03 & 243.34 & 236.22 & 236.22 & 236.22 & 29.63 & 29.52 & 29.58 & 242.26 & 243.26 & 242.76 \\
\hline $\mathrm{N}_{12}$ & 248.02 & 244.02 & 246.02 & 32.52 & 32.77 & 32.65 & 263.84 & 265.20 & 264.52 & 254.89 & 267.55 & 261.22 & 33.02 & 32.64 & 32.83 & 265.34 & 266.70 & 266.02 & 251.45 & 255.79 & 253.62 & 32.77 & 32.70 & 32.74 & 264.59 & 265.95 & 265.27 \\
\hline $\mathrm{N}_{13}$ & 264.59 & 265.42 & 265.01 & 38.08 & 36.86 & 37.47 & 276.92 & 278.28 & 277.60 & 277.96 & 277.79 & 277.87 & 38.24 & 38.36 & 38.30 & 278.42 & 279.78 & 279.10 & 271.27 & 271.61 & 271.44 & 38.16 & 37.61 & 37.88 & 277.67 & 279.03 & 278.35 \\
\hline $\mathrm{N}_{14}$ & 242.96 & 242.62 & 242.79 & 37.17 & 32.45 & 34.81 & 263.34 & 265.64 & 264.49 & 251.16 & 262.49 & 256.82 & 34.00 & 32.62 & 33.31 & 264.84 & 267.14 & 265.99 & 247.06 & 252.56 & 249.81 & 35.58 & 32.53 & 34.06 & 264.09 & 266.39 & 265.24 \\
\hline$N_{15}$ & 244.72 & 243.72 & 244.22 & 32.34 & 31.83 & 32.09 & 264.65 & 267.68 & 266.17 & 252.92 & 255.59 & 254.26 & 32.94 & 31.67 & 32.30 & 265.52 & 267.82 & 266.67 & 248.82 & 249.66 & 249.24 & 32.64 & 31.75 & 32.19 & 265.08 & 267.75 & 266.42 \\
\hline$N_{16}$ & 236.03 & 234.03 & 235.03 & 30.14 & 29.73 & 29.93 & 243.19 & 244.49 & 243.84 & 241.56 & 243.56 & 242.56 & 29.30 & 30.57 & 29.93 & 243.02 & 245.99 & 244.51 & 238.80 & 238.80 & 238.80 & 29.72 & 30.15 & 29.93 & 243.11 & 245.24 & 244.17 \\
\hline \multirow[t]{2}{*}{ MEAN } & 249.55 & 245.96 & & 33.31 & 33.78 & & 256.91 & 259.80 & & 252.93 & 259.33 & & 33.92 & 34.49 & & 258.33 & 262.41 & & 251.24 & 252.64 & & 33.62 & 34.13 & & 257.62 & 261.11 & \\
\hline & $\begin{array}{c}\text { S.Em } \\
\pm\end{array}$ & \multicolumn{2}{|c|}{ C.D at $5 \%$} & $\begin{array}{c}\text { S.Em } \\
\pm\end{array}$ & \multicolumn{2}{|c|}{ C.D at $5 \%$} & $\begin{array}{c}\text { S.Em } \\
\pm\end{array}$ & \multicolumn{2}{|c|}{ C.D at $5 \%$} & $\begin{array}{c}\text { S.Em } \\
\pm\end{array}$ & \multicolumn{2}{|c|}{ C.D at $5 \%$} & $\begin{array}{c}\text { S.Em } \\
\pm\end{array}$ & \multicolumn{2}{|c|}{ C.D at $5 \%$} & $\begin{array}{c}\text { S.Em } \\
\pm\end{array}$ & \multicolumn{2}{|c|}{ C.D at $5 \%$} & $\begin{array}{c}\text { S.Em } \\
\pm\end{array}$ & \multicolumn{2}{|c|}{ C.D at $5 \%$} & $\begin{array}{c}\text { S.Em } \\
\pm\end{array}$ & \multicolumn{2}{|c|}{ C.D at $5 \%$} & $\begin{array}{c}\text { S.Em } \\
\pm\end{array}$ & & \\
\hline Varieties & 0.54 & & & 0.59 & & is & 0.67 & & s & 0.44 & & 35 & 0.24 & & & 0.30 & & & 0.33 & 1 & & 0.41 & 1 & & 0.19 & & 60 \\
\hline Nutrients & 1.78 & & & 0.69 & & 97 & 1.95 & & 54 & 2.14 & & 05 & 0.56 & & 77 & 2.11 & & & 1.61 & & & 0.49 & & & 1.77 & & 02 \\
\hline$N$ at same & 2.52 & & & 0.98 & & is & 2.77 & & s & 3.02 & & s & 0.79 & & & 2.98 & & & 2.28 & $\mathrm{r}$ & & 0.69 & & & 2.51 & & s \\
\hline $\begin{array}{c}\text { Vat same } \\
\text { or different } \\
\quad \mathrm{N}\end{array}$ & 2.18 & & & 2.30 & & is & 2.71 & & s & 1.87 & & s & 0.97 & & s & 1.46 & & & 1.40 & s & & 1.62 & & & 0.98 & & s \\
\hline
\end{tabular}


Table.3 Uptake of N, P and $\mathrm{K}\left(\mathrm{kg} \mathrm{ha}^{-1}\right)$ as influenced by Isabgol varieties and integrated nutrient management

\begin{tabular}{|c|c|c|c|c|c|c|c|c|c|c|c|c|c|c|c|c|c|c|c|c|c|c|c|c|c|c|c|}
\hline \multirow{3}{*}{$\begin{array}{c}\text { Varieties Nutrients } \\
\text { Nutrients }\end{array}$} & \multicolumn{9}{|c|}{2015} & \multicolumn{9}{|c|}{2016} & \multicolumn{9}{|c|}{ Pooled } \\
\hline & \multicolumn{3}{|c|}{ Uptake $\mathbf{N}$} & \multicolumn{3}{|c|}{ Uptake $\mathrm{P}_{2} \mathrm{O}_{\mathrm{s}}$} & \multicolumn{3}{|c|}{ Uptake $\mathrm{K}_{2} \mathrm{O}$} & \multicolumn{3}{|c|}{ Uptake $\mathbf{N}$} & \multicolumn{3}{|c|}{ Uptake $\mathrm{P}_{2} \mathrm{O}_{5}$} & \multicolumn{3}{|c|}{ Uptake $\mathrm{K}_{2} \mathrm{O}$} & \multicolumn{3}{|c|}{ Uptake N } & \multicolumn{3}{|c|}{ Uptake $\mathrm{P}_{2} \mathrm{O}_{\mathrm{s}}$} & \multicolumn{3}{|c|}{ Uptake $\mathrm{K}_{2} \mathrm{O}$} \\
\hline & $\mathbf{v}_{1}$ & $\mathbf{V}_{2}$ & Mean & $\mathbf{v}_{1}$ & $\mathbf{v}_{2}$ & Mean & $\mathbf{V}_{1}$ & $\mathbf{V}_{2}$ & Mean & $\mathbf{v}_{1}$ & $\mathbf{V}_{2}$ & Mean & $\mathbf{V}_{1}$ & $V_{2}$ & Mean & $\mathbf{v}_{1}$ & $\mathbf{v}_{2}$ & Mean & $\mathbf{v}_{1}$ & $\mathbf{v}_{2}$ & Mean & $\mathbf{V}_{1}$ & $V_{2}$ & Mean & $\mathbf{V}_{1}$ & $\mathbf{v}_{2}$ & Mean \\
\hline $\mathrm{N}_{\mathrm{i}}$ & 23.55 & 26.32 & 24.93 & 8.28 & 7.51 & 7.90 & 18.97 & 16.90 & 17.94 & 23.65 & 21.82 & 22.73 & 8.31 & 6.80 & 7.55 & 18.94 & 16.49 & 17.71 & 23.60 & 24.07 & 23.83 & 8.29 & 7.16 & 7.73 & 18.96 & 16.69 & 17.82 \\
\hline $\mathbf{N}_{2}$ & 26.31 & 19.44 & 22.87 & 7.66 & 5.02 & 6.34 & 19.52 & 15.84 & 17.68 & 24.77 & 20.24 & 22.51 & 6.89 & 4.90 & 5.90 & 20.16 & 14.38 & 17.27 & 25.54 & 19.84 & 22.69 & 7.28 & 4.96 & 6.12 & 19.84 & 15.11 & 17.48 \\
\hline $\mathbf{N}_{3}$ & 22.66 & 20.50 & 21.58 & 6.84 & 5.75 & 6.30 & 16.30 & 12.37 & 14.34 & 23.19 & 21.14 & 22.17 & 6.94 & 7.32 & 7.13 & 19.94 & 16.31 & 18.12 & 22.92 & 20.82 & 21.87 & 6.89 & 6.54 & 6.72 & 18.12 & 14.34 & 16.23 \\
\hline $\mathrm{N}_{\star}$ & 35.11 & 33.25 & 34.18 & 11.64 & 10.77 & 11.20 & 25.95 & 24.29 & 25.12 & 34.59 & 31.99 & 33.29 & 12.45 & 12.23 & 12.34 & 26.35 & 26.30 & 26.33 & 34.85 & 32.62 & 33.73 & 12.04 & 11.50 & 11.77 & 26.15 & 25.29 & 25.72 \\
\hline $\mathbf{N}_{\mathrm{s}}$ & 24.23 & 18.93 & 21.58 & 8.68 & 7.26 & 7.97 & 16.15 & 15.98 & 16.06 & 25.95 & 21.58 & 23.77 & 8.82 & 6.88 & 7.85 & 18.11 & 16.52 & 17.31 & 25.09 & 20.25 & 22.67 & 8.75 & 7.07 & 7.91 & 17.13 & 16.25 & 16.69 \\
\hline $\mathrm{N}_{6}$ & 33.51 & 33.25 & 33.38 & 11.50 & 11.06 & 11.28 & 26.12 & 25.37 & 25.75 & 33.34 & 32.99 & 33.17 & 12.52 & 12.28 & 12.40 & 26.98 & 25.17 & 26.07 & 33.43 & 33.12 & 33.27 & 12.01 & 11.67 & 11.84 & 26.55 & 25.27 & 25.91 \\
\hline $\mathbf{N}_{7}$ & 22.27 & 14.55 & 18.41 & 6.48 & 3.60 & 5.04 & 13.01 & 10.39 & 11.70 & 18.89 & 16.01 & 17.45 & 6.61 & 5.02 & 5.82 & 15.45 & 10.77 & 13.11 & 20.58 & 15.28 & 17.93 & 6.55 & 4.31 & 5.43 & 14.23 & 10.58 & 12.41 \\
\hline $\mathrm{N}_{8}$ & 22.10 & 15.15 & 18.63 & 7.12 & 4.07 & 5.60 & 13.87 & 11.16 & 12.52 & 19.63 & 16.15 & 17.89 & 7.41 & 6.09 & 6.75 & 15.01 & 11.95 & 13.48 & 20.87 & 15.65 & 18.26 & 7.26 & 5.08 & 6.17 & 14.44 & 11.56 & 13.00 \\
\hline No & 24.74 & 13.03 & 18.88 & 6.82 & 4.05 & 5.43 & 16.15 & 10.52 & 13.34 & 19.96 & 14.70 & 17.33 & 7.38 & 5.18 & 6.28 & 16.97 & 11.91 & 14.44 & 22.35 & 13.86 & 18.11 & 7.10 & 4.62 & 5.86 & 16.56 & 11.21 & 13.89 \\
\hline$N_{10}$ & 19.98 & 17.08 & 18.53 & 8.52 & 4.04 & 6.28 & 18.50 & 10.68 & 14.59 & 16.54 & 14.21 & 15.38 & 5.99 & 5.44 & 5.71 & 18.11 & 11.40 & 14.75 & 18.26 & 15.64 & 16.95 & 7.25 & 4.74 & 6.00 & 18.30 & 11.04 & 14.67 \\
\hline $\mathrm{N}_{\mathrm{N}}$ & 35.74 & 35.18 & 35.46 & 11.78 & 12.11 & 11.95 & 28.43 & 24.84 & 26.64 & 35.81 & 35.52 & 35.67 & 13.50 & 12.57 & 13.03 & 28.07 & 25.75 & 26.91 & 35.78 & 35.35 & 35.56 & 12.64 & 12.34 & 12.49 & 28.25 & 25.30 & 26.77 \\
\hline$N_{12}$ & 17.08 & 15.20 & 16.14 & 6.60 & 4.55 & 5.57 & 13.87 & 9.54 & 11.71 & 16.21 & 14.69 & 15.45 & 8.18 & 4.91 & 6.55 & 13.47 & 10.60 & 12.03 & 16.64 & 14.95 & 15.80 & 7.39 & 4.73 & 6.06 & 13.67 & 10.07 & 11.87 \\
\hline$N_{13}$ & 13.71 & 9.87 & 11.79 & 4.33 & 3.87 & 4.10 & 5.94 & 5.46 & 5.70 & 12.75 & 9.56 & 11.15 & 4.63 & 3.80 & 4.22 & 8.07 & 6.57 & 7.32 & 13.23 & 9.72 & 11.47 & 4.48 & 3.83 & 4.16 & 7.01 & 6.02 & 6.51 \\
\hline$N_{14}$ & 16.87 & 13.76 & 15.32 & 5.65 & 4.03 & 4.84 & 9.76 & 7.90 & 8.83 & 15.50 & 12.20 & 13.85 & 6.92 & 5.06 & 5.99 & 11.44 & 7.98 & 9.71 & 16.19 & 12.98 & 14.58 & 6.28 & 4.54 & 5.41 & 10.60 & 7.94 & 9.27 \\
\hline$N_{15}$ & 14.31 & 10.77 & 12.54 & 5.60 & 3.94 & 4.77 & 6.49 & 5.15 & 5.82 & 14.99 & 10.34 & 12.66 & 9.51 & 4.84 & 7.18 & 10.93 & 8.52 & 9.72 & 14.65 & 10.55 & 12.60 & 7.55 & 4.39 & 5.97 & 8.71 & 6.83 & 7.77 \\
\hline$N_{16}$ & 34.98 & 31.13 & 33.06 & 12.19 & 10.73 & 11.46 & 26.53 & 24.38 & 25.46 & 34.99 & 29.86 & 32.43 & 12.12 & 11.59 & 11.86 & 26.71 & 25.83 & 26.27 & 34.98 & 30.50 & 32.74 & 12.16 & 11.16 & 11.66 & 26.62 & 25.10 & 25.86 \\
\hline \multirow[t]{2}{*}{ Mean } & 24.20 & 20.46 & & 8.10 & 6.40 & & 17.22 & 14.42 & & 23.17 & 20.19 & & 8.64 & 7.18 & & 18.42 & 15.40 & & 23.68 & 20.33 & & 8.37 & 6.79 & & 17.82 & 14.91 & \\
\hline & S.Em \pm & \multicolumn{2}{|c|}{ C.D at $5 \%$} & $\begin{array}{c}\text { S.Em } \\
\pm\end{array}$ & \multicolumn{2}{|c|}{ C.D at $5 \%$} & $\begin{array}{c}\text { S.Em } \\
\pm\end{array}$ & \multicolumn{2}{|c|}{ C.D at $5 \%$} & $\begin{array}{c}\text { S.Em } \\
\pm\end{array}$ & \multicolumn{2}{|c|}{ C.D at $5 \%$} & $\begin{array}{c}\text { S.Em } \\
\pm\end{array}$ & \multicolumn{2}{|c|}{ C.D at $5 \%$} & $\begin{array}{c}\text { S.Em } \\
\pm\end{array}$ & \multicolumn{2}{|c|}{ C.D at $5 \%$} & $\begin{array}{l}\text { S.Em } \\
\pm\end{array}$ & \multicolumn{2}{|c|}{ C.D at $5 \%$} & $\begin{array}{c}\text { S.Em } \\
\pm\end{array}$ & \multicolumn{2}{|c|}{ C.D at $5 \%$} & $\begin{array}{l}\text { S.Em } \\
\pm\end{array}$ & \multicolumn{2}{|c|}{ C.D at $5 \%$} \\
\hline Varieties (V) & 0.02 & & .80 & 0.11 & & & 0.05 & & & 0.38 & & 16 & 0.28 & & & 0.21 & 0.6 & & 0.20 & & .62 & 0.16 & & & 0.13 & & 41 \\
\hline Nutrients (N) & 1.29 & & .65 & 0.49 & & & 0.88 & & & 0.77 & & 20 & 0.48 & & & 0.82 & 2.3 & & 0.86 & & .45 & 0.36 & & & 0.66 & & 88 \\
\hline $\mathrm{N}$ at same $\mathrm{V}$ & 1.82 & & vs & 0.70 & & & 1.25 & & & 1.10 & & s & 0.69 & & & 1.17 & Ns & & 1.22 & & vs & 0.51 & & & 0.94 & & is \\
\hline Vat same or different $\mathrm{N}$ & 1.11 & & vs & 0.49 & & & 0.36 & & & 1.50 & & s & 1.10 & & & 0.89 & No & & 0.85 & & vs & 0.64 & & & 0.57 & & is \\
\hline
\end{tabular}

\begin{tabular}{|c|c|c|c|}
\hline $\mathrm{V}_{1}$-Vallabh Isabgol- 1 & $\mathrm{~V}_{2}$-Gujarat Isabgol-2 & $\mathrm{N}_{1}-$ RDF FYM + RDF NPK $\left(\mathrm{kg} \mathrm{h}^{-1}\right)$ & $\mathrm{N}_{2}-\mathrm{RDF} F \mathrm{FYM}+\mathrm{RDF} \mathrm{NPK+ZnSO4}$ \\
\hline $\mathrm{N}_{3}$ - RDF FYM + RDF NPK+FeSO4 & $\mathrm{N}_{4}-\mathrm{RDF} F \mathrm{FYM}+\mathrm{RDF} \mathrm{NPK}+\mathrm{ZnSO} 4+\mathrm{FeSO} 4$ & $\mathrm{~N}_{5}$-Vermicompost $\left(1 \mathrm{tha}^{-1}\right)+\mathrm{RDF}$ NPK & $\mathrm{N}_{6}$-Vermicompost + 50\% RDF NPK + ASP + AZF \\
\hline $\mathrm{N}_{-}-75 \%$ RDF FYM $+75 \%$ RDF NPK & $\mathrm{N}_{8}-75 \%$ RDF FYM + 75\% RDF NPK + AZB, & No- $75 \%$ RDF FYM + $75 \%$ RDF NPK + ASP & $\mathrm{N}_{\mathrm{l}-}-75 \%$ RDF FYM + $75 \%$ RDF NPK + PSB \\
\hline $\begin{array}{l}\mathrm{N}_{11}-75 \% \text { RDF FYM + } \\
\text { ZnSO4 + FeSO4 }\end{array}$ & $\mathrm{N}_{\mathrm{L}^{2}-} 50 \%$ RDF FYM $+50 \%$ RDF NPK & $\mathrm{N}_{\mathrm{l} 3}-50 \%$ RDF FYM + 50\% RDF NPK + AZB & $\mathrm{N}_{\mathrm{l}-}-50 \%$ RDF FYM + 50\% RDF NPK + ASP \\
\hline $\mathrm{N}_{15} 50 \%$ RDF FYM + 50\% RDF NPK + PSB & $50 \%$ RDF FYM + 75\% RDF NPK + ASP + PSB + ZnSO4 + FeSO4 & NS = Non-significant & DAS $=$ Days after sowing \\
\hline
\end{tabular}


The higher soil organic carbon (\%) may be due to use of different doses of integrated nutrient management application especially which helps to increase the organic carbon content in soil which is an indication of soil health and its productivity potential in soil by adding soil biomass carbon and its activity compared with mineral fertilizers. Microbial metabolic activity (dehydrogenase activity per microbial biomass organic carbon), which was significantly higher under balanced fertilization helps to increase uptake of more nutrients to plants and also have a profound effect on the improvement of soil structure (Singh et al.,1994 and Indra and Shivran, 2009) in Isabgol.

The available Nitrogen, Phosphorous and Potassium were significantly influenced by integrated nutrient management. The minimum available N P K was recorded with application of $75 \%$ RD of FYM $+75 \%$ RD of NPK + Azospirillum $+P S B+\mathrm{ZnSO}_{4}+$ $\mathrm{FeSO}_{4}\left(\mathrm{~N}_{11}\right)$ which was on par with $50 \% \mathrm{RD}$ of FYM + $75 \% \mathrm{RD}$ of NPK + Azospirillum + $P S B+\mathrm{ZnSO}_{4}+\mathrm{FeSO}_{4}\left(\mathrm{~N}_{16}\right), \mathrm{RD}$ of $\mathrm{FYM}+$ $\mathrm{RD}$ of $\mathrm{NPK}+\mathrm{ZnSO}_{4}+\mathrm{FeSO}_{4}\left(\mathrm{~N}_{4}\right)$ and Vermicompost $+50 \%$ RD of NPK + Azospirillum + Azotobacter $\left(\mathrm{N}_{6}\right)$ and maximum available NPK was recorded with application of $50 \%$ RD of FYM + $50 \%$ RD of NPK+Azotobacter $\left(\mathrm{N}_{13}\right)$ it was due to easy decomposition and mineralisation of nutrients leads to better uptake of nutrient to plant. Further higher yield response may be due to effective utilization of NPK nutrients from higher dose of inorganic fertilizers and organic manures which enhanced increased solubility of nutrients, nitrogen loss and increased the biological nitrogen fixation along with minimizing residual effect of applied nutrients and mineralization thus release of nutrients at faster rates helps for plant to more nutrients availability in soil, vermicompost along with dual application of nitrogen fixer and $P S B$ helps in fixation of atmospheric nitrogen and solubilizing unavailable P to available form. ( Shivran et $a l ., 2014$ and Venkatesh,2007).

The nutrient content and uptake of nitrogen, phosphorus and potassium were significantly higher was recorded with application of $75 \%$ $\mathrm{RD}$ of $\mathrm{FYM}+75 \% \mathrm{RD}$ of $\mathrm{NPK}+$ Azospirillum $+P S B+\mathrm{ZnSO}_{4}+\mathrm{FeSO}_{4}\left(\mathrm{~N}_{11}\right)$ which was on par with RD of FYM + RD of $\mathrm{NPK}+\mathrm{ZnSO}_{4}+\mathrm{FeSO}_{4}\left(\mathrm{~N}_{4}\right), 50 \% \mathrm{RD}$ of $\mathrm{FYM}+75 \% \mathrm{RD}$ of NPK + Azospirillum + $P S B+\mathrm{ZnSO}_{4}+\mathrm{FeSO}_{4}\left(\mathrm{~N}_{16}\right)$ and Vermicompost $+50 \%$ RD of NPK + Azospirillum + Azotobacter $\left(\mathrm{N}_{6}\right)$ and lower uptake of NPK was recorded with application of $50 \%$ RD of FYM + $50 \%$ RD of NPK + Azotobacter $\left(\mathrm{N}_{13}\right)$. Uptake of $\mathrm{N}, \mathrm{P}$ and $\mathrm{K}$ might have enhanced due to increased content of seed yield and straw yield, the available soil nitrogen increases with biofertilizer application which might have helped in better utilization of native nitrogen and also nitrogen fixation.

However, because of INM treatments helps release of nutrients faster rate, optimum nutrition supply, indicated significantly higher nutrient uptake under various INM modules, in this inorganic indicated unrestricted availability of these nutrients in labile pool to support the crop growth and yield. The positive soil $\mathrm{N}, \mathrm{P}$ and $\mathrm{K}$ balance observed in plots receiving organic manures, inorganic, biofertilizers these combinations may be attributed to the richness of these sources in terms of organic matter and enhanced microbial activity. Since the uptake of nutrient is a function of concentration finally accelerates the increase in seed yield and straw yield. All fertilizing treatments caused a significant increase in seed yield however manure was effective in improving soil porosity and plant tolerance to heavy metals, (Choudhary and Shivran, 2009 and Mirshekari and Forouzandeh, 2015). Non 
significant interaction effect was observed with EC, $\mathrm{pH}, \mathrm{OC}$, nutrient content of available N P K and Uptake of N P K.

\section{References}

Choudhary, I. and Shivran, A. C., 2009, Effect of integrated nutrient management on yield quality and nutrient uptake of Isabgol. Ann. Agric. Res. New Series, 30 (3 and 4): 105-107.

Choudhary, T., Sharma, S. K. and Yadav, B. K., 2014, Influence of FYM and inorganic fertilizers on growth and yield of Isabgol (Plantago ovata Forsk.). J. Spices Aromatic Crops, 23 (1): 130-136.

Goraya, G.S. and Ved, D. K., 2019, Medicinal plants in India: An assessment of their demand and supply, NMPB, Ministry of AYUSH Government of India and ICFRE Dehradun.

Jackson, M.L., 1973, Soil chemical analysis. Prentice hall of India private limited, New Delhi, pp. 485.

Kumar, S. R., Beniwal., B. R. and Choudhary. B. R., 2009, A high yielding Isabgol (Plantago ovata Forsk.) genotype for arid western plain of Rajasthan. Annals of Arid Zone, 48(2): 169-170.

Kumar, M., Jakhar, M. S. and Singh, V. P., 2015, Effect of varying nitrogen levels on growth and yield of Isabgol (Plantago ovata Forsk.). Annals of Horticulture, 8 (2): 212-214.

Nadim, M, M., Ahmad, J., Umer, S. and Bakshi, S.K., 2011, Influence of nutrients and microorganisms on the growth and yield of (Plantago ovata Forsk.). Trends in Biosciences, 4 (2): 169-171.

Nadukeri, S., Kattimani, K. N. and Kolakar, S. S., 2014, Influence of organic and inorganic fertilizers on growth and tuber yield of Coleus (Coleus forskohlii Briq.) under northern dry zone of Karnataka. Intl. J. Agric. Sci., 10 (1): 119-123.

Panse, V. G. and Sukhatme, P. V., 1983,
Statistical Methods for Agric. Workers. Indian Council of Agric. Res., New Delhi, pp. 152-174.

Piper, C. S., 1966, Soil and plant analysis inter sciences publications, Inc., New York.

Repsiene, R., 2001, Efficiency of placement fertilization for barley yield in conservation agriculture a worldwide Challenge. First World Congress on Conservation agriculture; Madrid, Spain, 2: 327-330.

Solanki, K. S., Singh, R. R. and Chauhan, R. S., 1987, Effect of nitrogen, agrochemicals and azotobacter inoculation with and without FYM on yield and quality of rainfed Barley. Indian J. Agril. Res., 21 (2): 83-87.

Saxena, A. and Rao, A. V., 2000, Response of Isabgol to aztobacter inoculation under field condation in arid zone. Annals of Arid Zone, 39(2): 199-201.

Shivran, A. C., Choudhary, I. and Puniya, M. M., 2014, Influence of integrated nutrient management and biofertilizer on growth yield and soil fertility of blond Psyllium (Plantago ovata Forsk.) cultivation under semi-arid conditions. Ann. Agric. Res., New Series. 35 (4): 379-385.

Shivran, A. C. and Jat, N. L., 2015, Integrated nutrient management influenced growth yield and economics of Fennel (Foeniculum vulgare) under semi-arid conditions. Ind. J. Agronomy, 60 (2): 318323.

Shivran, A. C., 2016a, Response of bond Psyllium (Plantago ovata Forsk.) varieties to time of sowing and nitrogen fertilization under semi-arid condition. Intl. J. Seed Spices., 6 (2): 50-54.

Shivran, A. C., 2016b, Growth yield and nutrient uptake of Isabgol (Plantago ovata Forsk.) with phosphorus PSB and zinc fertilization. Intl. J. Seed Spices, 6 (1): 6673.

Shivran, A. C., Choudhary, I. and Puniya, M. 
M., 2014, Influence of integrated nutrient management and biofertilizer on growth yield and soil fertility of blond Psyllium (Plantago ovata Forsk.) cultivation under semi-arid conditions. Ann. Agric. Res., New Series. 35 (4): 379-385.

Singh., S.B., Chauhan. and Vishwanath, 2011, Evaluation of Isabgol (Plantago ovata Forsk.) varieties to potash levels in semiarid condition. The J. Rural \& Agric. Res., (2): 58-59.

Stanford, S. and English., 1963, Use of flame photometer in rapid soils tests for potassium and calcium. Agronomy. J., 41: 446-447.

Tripathi, V. K., Sanjeev Kumar, Katiyar, P. N. and Nayyer, M.A., 2013, Integrated nutrient management in Isabgol (Plantago ovata Forsk.). Progressive Horticulture, 45: 302-305.

Subbaiah, B.V. and Asija, G.L., 1956, A rapid procedure for determination of available nitrogen in soils. Current. Sci., 25: 259260.

Olsen, S. R., Col, S. C. W., Watanabe, P. S. and Dean, L. A., 1954, Estimation of available $\mathrm{P}$ in soil by extraction with $\mathrm{HNO}_{3}$, Circular USDA. pp. 931.

Sharma and Ratnoo., 2013, Isabgol crop yield enhancement in transitional plain of luni basin of Rajasthan through seed and fertilizer management practices, www.Indian Journals.com.

Venkatesh, N.T. 2007, Integrated nutrient management in Isabgol (Plantago ovate Forsk.). M.Sc (Horti)Thesis, UAS Dharwad.

Kazal, V., Bhushan, B. Kumari, R., Narwal. K, S. and Pal, A., 2017, Evaluation of salt tolerance in different Isabgol (Plantago ovata Forsk.) genotypes under differential salt stress imposition. Indian J. Agric. Biochemistry., 30 (1): 41-49.

Mirshekari, S. and Forouzandeh, M., 2015, Analysis of effect of organic fertilizer on yield and active substance of Psyllium herbal plant (Plantago ovata L.). Biological Forum - An Intl. J., 7(1): 436440

\section{How to cite this article:}

Siddalingayya V. Salimath, K. N. Kattimani, Y. K. Kotikal, N. S. Nagarja, Jameel Jhalegar, D. R. Patil and Venkatesh, J. 2020. Response of Mode of Application with Integrated Nutrient Management on soil parameters of Isabgol (Plantago ovata Forsk.) under Northern Dry Zone of Karnataka. Int.J.Curr.Microbiol.App.Sci. 9(12): 2952-2962.

doi: https://doi.org/10.20546/ijcmas.2020.912.350 